\title{
Local magnetic divertor for control of the plasma-limiter interaction in a tokamak
}

\author{
S. J. Zweben, P. C. Liewer, and R. W. Gould \\ Department of Applied Physics, California Institute of Technology, Pasadena, California 91125
}

(Received 14 January 1983; accepted 5 December 1983)

\begin{abstract}
An experiment is described in which plasma flow to a tokamak limiter is controlled through the use of a local toroidal divertor coil mounted inside the limiter itself. This coil produces a local perturbed field $B_{C}$ approximately equal to the local unperturbed toroidal field $B_{T} \cong 3 \mathrm{kG}$, such that when $B_{C}$ adds to $B_{T}$ the field lines move into the limiter and the local plasma flow to it increases by a factor as great as 1.6 , and when $B_{C}$ subtracts from $B_{T}$ the field lines move away from the limiter and the local plasma flow to it decreases by as much as a factor of 4 . A simple theoretical model is used to interpret these results. Since these changes occur without significantly affecting global plasma confinement, such a control scheme may be useful for optimizing the performance of pumped limiters.
\end{abstract}

\section{INTRODUCTION}

Recently the use of mechanical divertors for heat and particle removal in large tokamaks has been proposed. ${ }^{1}$ The structure of these "pumped limiters" will depend to a large extent upon the cross-field particle and heat flows which occur in the turbulent tokamak boundary layer. ${ }^{2,3}$ Unfortunately, the magnitude of the cross-field diffusion is variable in practice ${ }^{3,4}$ and presently difficult to calculate in theory, 5,6 thus introducing significant uncertainties into pumped limiter design. There is clear evidence from recent experiments ${ }^{7-10}$ that prototype pumped limiter designs work successfully in moderate-sized tokamaks. However, given the very large heat load expected in reactor-relevent pumped limiter applications, it is likely that in the future it will be necessary to have some direct or active control over the plasma-limiter contact in order to avoid heat-induced damage to these limiters' leading edge.

Ordinarily, the only active control of the plasma-limiter contact in divertorless tokamaks is through plasma repositioning or neutral gas puffing at the boundary. This type of control is generally not fast enough or sure enough to avoid occasional structural damage to present-day tokamak limiters. ${ }^{11}$ Of course, such control has also been achieved through multiple or single-null poloidal and bundle divertors which remove the plasma-wall interaction to a remote divertor plate, a solution the complexity and cost of which is supposed to be avoided by the pumped limiter proposals.

In this paper we describe an experiment which shows how active control of plasma flow to a limiter can be made by using a local toroidal divertor coil mounted inside the limiter itself. Here, a small rectangular coil inside the limiter is oriented so that the coil's field either adds to or subtracts from the main toroidal field $B_{T}$. When the coil's field $B_{C}$ adds to $B_{T}$, field lines are pulled out of the plasma into the limiter, and the plasma flow to the limiter is observed to increase by up to a factor of 1.6. When the coil's field subtracts from $B_{T}$, field lines are pushed out around the limiter, and the plasma flow to the limiter is observed to decrease by up to a factor of 4. These changes in plasma-limiter contact are obtained without significant changes in global plasma confinement.
These experimental results on plasma flow to the limiter are compared to theoretical predictions from a simple model. First the trajectories of the magnetic field lines which intersect the limiter are calculated for various divertor fields; the expected plasma flux to the limiter is then calculated by assuming that the plasma incident into the divertor region is diverted along these field lines. For the case in which the divertor field adds to the local toroidal field, the local plasma flux (particles $/ \mathrm{cm}^{2} \mathrm{sec}$ ) along a field line is increased near the divertor due to the compression of the magnetic flux; however, in this case some of the incident particles are reflected by the divertor field's mirror. For the case in which the divertor field subtracts from the local toroidal field, the local plasma flux along a field line is decreased due to the expansion of the magnetic flux; there is no mirroring in this case. The inclusion of a constant poloidal field into this model does not significantly change these results. Reasonably good agreement is found between this simple model and the experimental data.

In Sec. II, the experimental configuration is described. Experimental results are presented in Sec. III, and in Sec. IV, model magnetic divertor calculations are presented and compared with the experimental results. In Sec. V, the comparison is evaluated and some additional effects not included in the model are discussed. In Sec. VI we give the conclusions.

\section{EXPERIMENTAL CONFIGURATION}

\section{A. Tokamak and limiter coil design}

For this experiment the Caltech tokamak ${ }^{12}$ had the following parameters: toroidal field $B_{T}=3.6 \mathrm{kG}$ on axis, plasma current $I=20 \mathrm{kA}$, major and minor radii $R=45 \mathrm{~cm}$ and $a=16 \mathrm{~cm}$, respectively, line averaged density $\bar{n}=0.2-$ $1.0 \times 10^{13} \mathrm{~cm}^{-3}$, and central electron temperature (assuming Spitzer conductivity at $Z=2$ ) of $T_{e} \cong 100 \mathrm{eV}$. For a typical one-turn loop voltage of $2 \mathrm{~V}$ at a peak current of $20 \mathrm{kA}$ with $\bar{n}=5 \times 10^{12} \mathrm{~cm}^{-3}$, the global energy confinement time is approximately $0.5 \mathrm{msec}$, while the pulse length is typically $10-15 \mathrm{msec}$. The machine is normally operated without any 
(a)

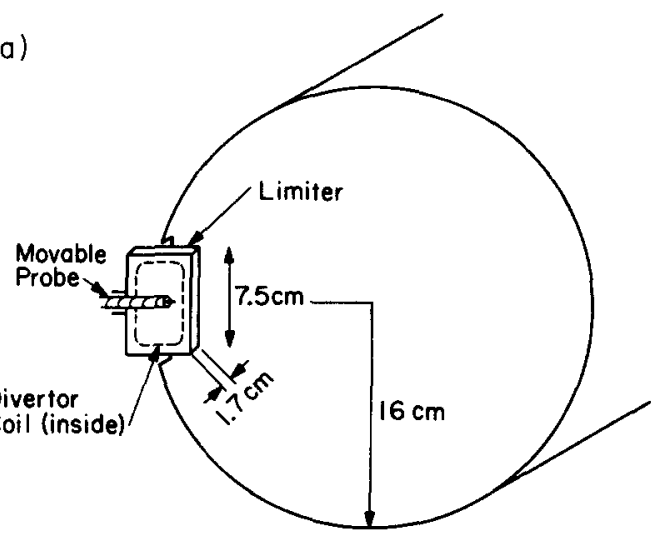

(b)

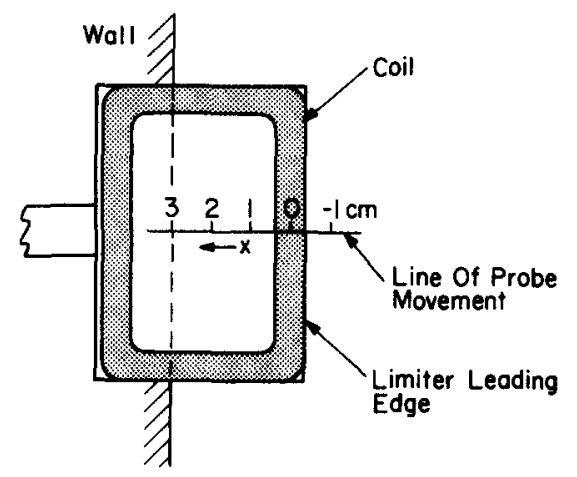

FIG. 1. Geometry of the tokamak and of the local divertor coil inside the outer limiter. In (a) is shown the placement of the outer limiter in the chamber, with the divertor coil inside the limiter (dashed line) and the movable Langmuir probe adjacent to it. In (b) is shown the limiter and coil construction, including the radial coordinate system used to plot the results.

localized limiters, and the walls are routinely Taylor discharge-cleaned similarly to the UCLA tokamaks. ${ }^{13}$ The overall plasma impurity level is monitored by a broadband uv detector ${ }^{14}$ which indicates that a relatively clean plasma can be obtained $(Z \cong 2)$.

For these experiments a small radially movable limiter was inserted at the outer equatorial plane as shown in Fig. 1(a). The limiter surfaces which contact the plasma are made of $0.3 \mathrm{~cm}$ thick stainless steel which is discharge-cleaned along with the walls before operating the machine. The insertion of a limiter such as this has several effects on the plasma; it can decrease the overall plasma current due to a reduction of the effective minor radius of the column; it generally increases the hydrogen recycling coefficient such that less external gas feed is needed to obtain a given plasma density; it lowers the maximum density which can be obtained before disruption; and it also somewhat increases the impurity level as monitored by the uv detector. These effects are relatively small for an insertion distance of $3 \mathrm{~cm}$ as used in this experiment, i.e., the maximum plasma current at a given average density is reduced by $\leqslant 20 \%$ with the insertion of the limiter, the total uv level is increased by less than a factor of 2 , and the maximum density at $q(a) \cong 4$ is decreased by less than $50 \%$. Other than this the discharge with a limiter behaves globally quite similarly to limiterless discharges.

Inside the $7.5 \mathrm{~cm}$ high by $1.7 \mathrm{~cm}$ wide outer limiter used for this experiment is an eight-turn Teflon-insulated coil which is used to produce the local field perturbation. A side view of the limiter is shown approximately to scale in Fig. 1(b). The coil is fixed in the plane of the limiter such that it produces through its center a field $B_{C}$ which either adds to or subtracts from the local toroidal field.

Some typical magnetic field configurations are shown in Fig. 2. In 2(a) is the coordinate system: the coil is modeled by a rectangular loop located in the $x-y$ plane, and the (unperturbed) toroidal field is in the $z$ direction. The actual coil dimensions are $a=2.2 \mathrm{~cm}$ and $b=3.2 \mathrm{~cm}$. The $x$ direction corresponds to the usual radial direction through the minor axis of the tokamak; however, $x=0$ is defined so as to pass

(a)

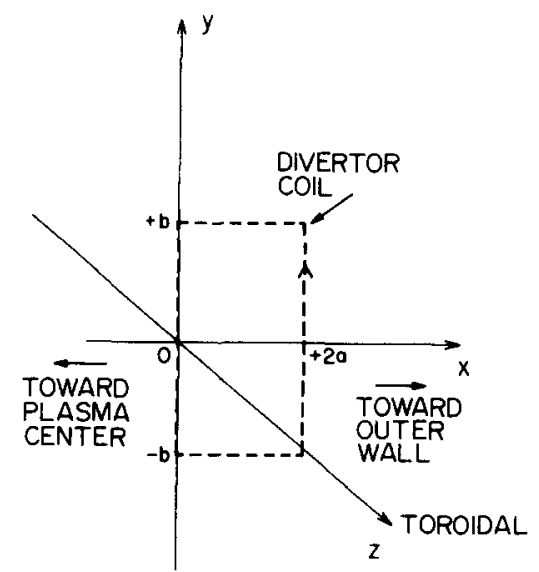

(b)

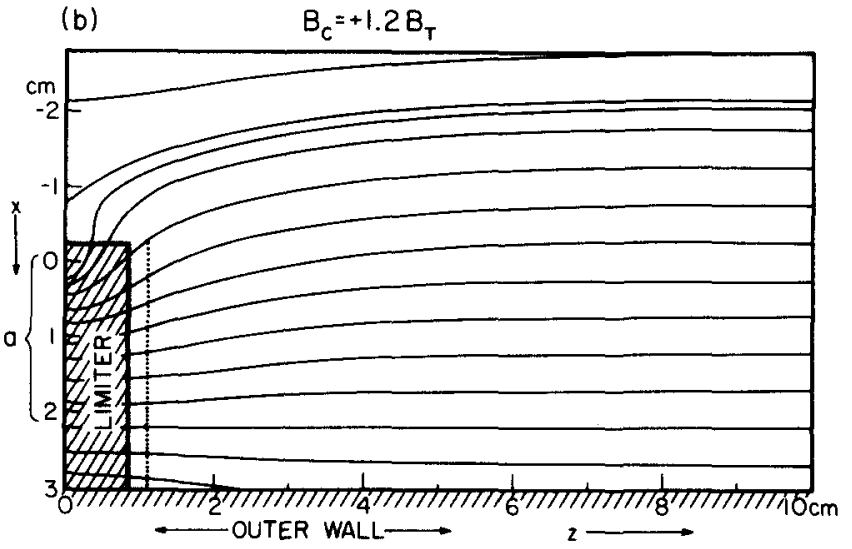

(c) $B_{c}=-1.2 B_{T}$

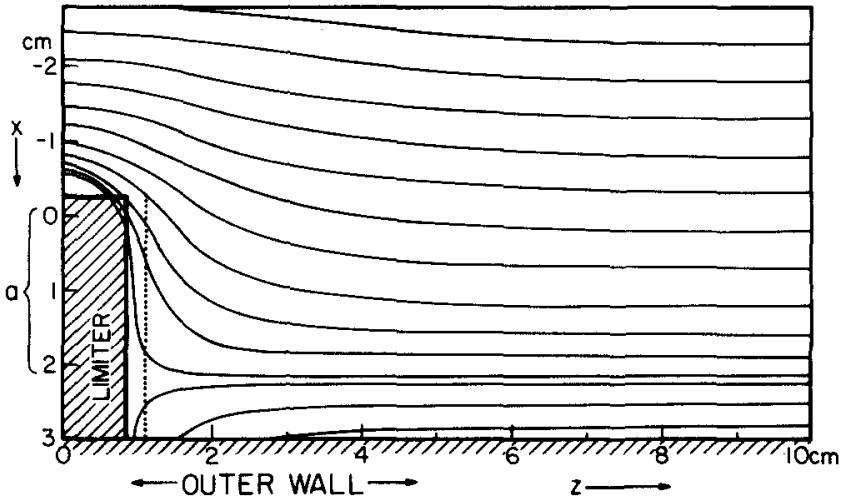

FIG. 2. Magnetic field patterns calculated for the local divertor in the vicinity of the limiter. In (a) is the coordinate system, where for our case $a=2.2$ $\mathrm{cm}$ and $b=3.2 \mathrm{~cm}$. In (b) and (c) are the field line patterns for the cases $B_{C}=+1.2 B_{T}$ and $B_{C}=-1.2 B_{T}$, respectively, which correspond to the usual experimental configurations. The line of movement of the probe is shown by the dotted line adjacent to the limiter surface. 
through the center of the vertical coil segment nearest to the plasma, and $\boldsymbol{x}$ increases toward the outer chamber wall (see also Fig. 1).

In Figs. 2(b) and 2(c) are numerically computed magnetic field line plots which show the coil's field added to a uniform toroidal field, where $B_{C}= \pm 1.2 B_{T}$ for these cases. The strength of the coil's field $B_{C}$ and toroidal field $B_{T}$ will always be that measured at the geometrical center of the coil, i.e., at $x=2.2 \mathrm{~cm}, y=0, z=0$ in these figures. These plots show the radial and toroidal structure of the perturbed fields as viewed from the top of the machine, including the appropriate limiter and wall geometry corresponding to the experimental configuration. Note that the vertical coil segment nearest to the plasma is centered at $x=0, z=0$, and that a part of the limiter structure extends past this coil segment to $x=-0.25 \mathrm{~cm}$.

When the coil's field adds to the toroidal field as in 2(b), the field lines are pulled in towards the limiter, and when the coil's field opposes the toroidal field as in 2(c), the field lines are pushed out and away from the limiter. The range of field perturbations used in this experiment was $B_{C}= \pm 0.8-1.8$. $B_{T}$; however, the most detailed results are obtained for the cases $B_{C}= \pm 1.2 B_{T}$, which correspond to the field patterns shown in Fig. 2.

The limiter coil is energized during the middle of a tokamak discharge with a limiter coil current rise time of $1 \mathrm{msec}$ and a maximum coil current of $20 \mathrm{kA}$ turns. The field penetration time through the stainless steel limiter body is less than $0.5 \mathrm{msec}$, and the limiter coil current decay time is 8 msec. Thus the perturbed field can be applied after the initial breakdown and current-rise phases of the discharge, and it remains on at nearly full field for most of the flat-current phase of the discharge (see Fig. 3). After a run of typically 100 tokamak discharges, the temperature of the limiter structure equilibrates at about $50^{\circ} \mathrm{C}-100^{\circ} \mathrm{C}$, which is low enough to avoid significant impurity generation from outgassing. The stainless steel limiter body is locally grounded to the chamber wall for all of these measurements.

Mechanical torques have not been a problem in the operation of this divertor, since the coil lies in the plane perpendicular to the toroidal field and the main forces involve either a compression or expansion which is taken up by the body of the limiter itself. There is a net radial force due to the interaction of the coil's magnetic moment with the $1 / R$ toroidal field; however, at these low fields this force can easily be restrained at the flange which holds the vacuum seal for the limiter mount.

\section{B. Edge plasma dlagnostics}

All of the data concerning the effect of this local toroidal divertor on the plasma-limiter interaction have been obtained using Langmuir probes; mainly a single-tipped 0.03 $\mathrm{cm}$ diam, $0.2 \mathrm{~cm}$ long probe which scans along the equatorial plane $0.25 \mathrm{~cm}$ away from the ion-drift side of the limiter [see Figs. 1(a), 2(b), and 2(c)]. This probe was placed as close as possible to the limiter surface in order for the probe measurements to be representative of the plasma which is intersecting the limiter. All theoretical calculations of the effects on plasma flow are referred to the probe positions and not to the
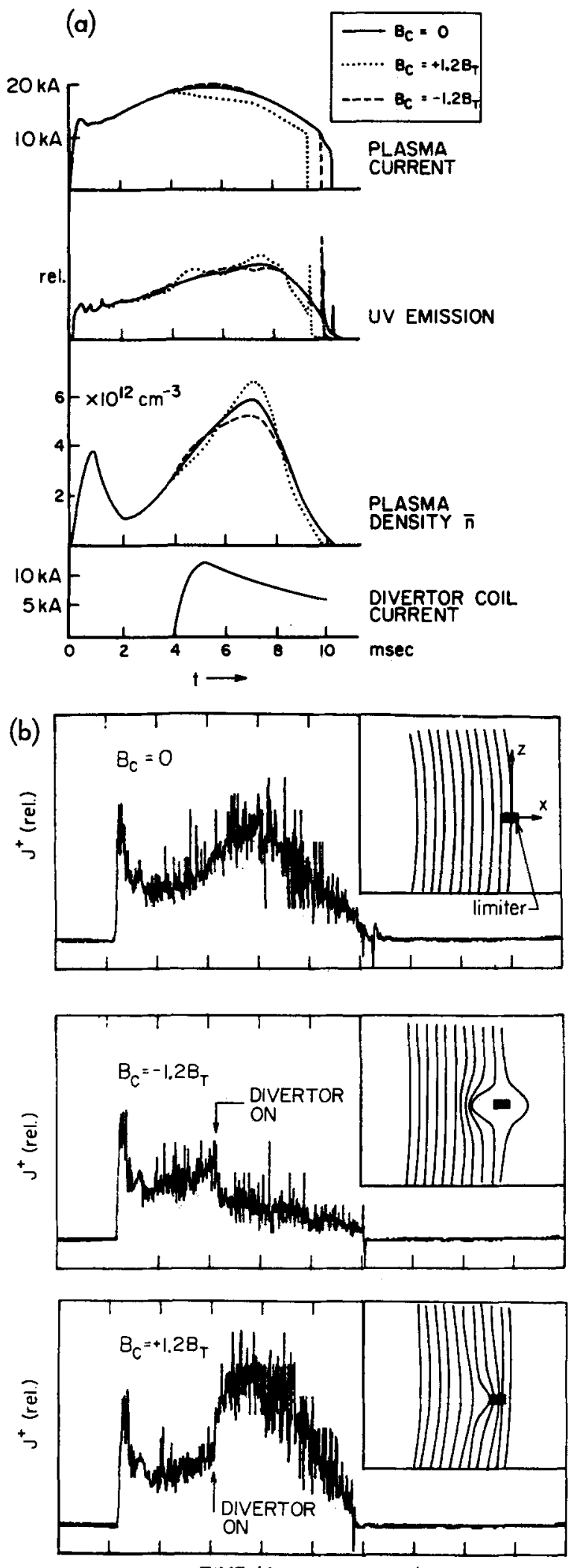

TIME (2 msec/division)

FIG. 3. Effects of the divertor on global and local plasma properties. Part (a) shows the effect of the activation of the divertor $(t=4 \mathrm{msec})$ on the plasma current, total ultraviolet light emission, and on the line averaged plasms density measured through the plasma center. These global plasma properties are not significantly affected by the divertor. In part (b) are ion saturation current signals $J^{+}$from the Langmuir probe adjacent to the limiter, for the same three discharges shown in part (a). The probe is located at $x=1 \mathrm{~cm}$ for these examples. Inserts show the calculatod magnetic field patterns for those cases. Activation of the divertor significantly changes the ion flux to this probe due to the local diversion of the magnetic field. 
adjacent limiter positions (discussion of plasma flow to the limiter itself is given in Sec. V D).

For this experiment we use the probe's ion saturation current $I^{+}$, or equivalently the current density $J^{+}=I^{+} / A$ (where $A$ is the probe area), as a measure of the incident ion flux at various positions directly adjacent to the limiter. The probe bias of $-100 \mathrm{~V}$ used here is sufficient to put the probe well into the ion saturation region of its $(I, V)$ characteristic (similar results are obtained over a range of probe biases of -100 to $-200 \mathrm{~V}$ ). Since for most purposes only the relative values of $J^{+}$are important, the uncertainty in the effective probe area $A$ does not directly affect the results.

In order to estimate the local density we use the approximate relation: $I^{+}=\frac{1}{4} n e \bar{v} A$, where $\bar{v}=\left(8 k T_{e} / m_{i} \pi\right)^{1 / 2}$ is a typical velocity for collected ions, ${ }^{4,15}$ and where we take $A$ to be the full geometrical probe area since the ion gyroradius here is larger than the probe radius. (This approximation is intended for the case in which $T_{i} \cong T_{e}$ and the directed ion velocity is subsonic.) In order to measure the electron temperature, we use an exponential fit of the probe's $(I, V)$ characteristic, while the space potential is estimated (with considerable uncertainty) as the "knee" of the same $(I, V)$ curve. ${ }^{16}$ The floating potential of the probe has also been measured as a function of time by a high impedance voltage monitor.

In inferring these plasma properties from the probe data, no explicit corrections have been made for nonisotropic distribution functions which might occur along with the divertor; for example, in one case particle mirroring will increase the perpendicular energy at the expense of the parallel energy. However, since the probe is cylindrical it is exposed to both parallel and perpendicular velocity components, so to a first approximation the probe results average over these two components.

Typical densities and temperatures measured behind this limiter are $n=10^{11} \mathrm{~cm}^{-3}$ to $10^{12} \mathrm{~cm}^{-3}$ and $T_{e}=5-25$ $\mathrm{eV}$. These values are consistent with those measured with Langmuir probes in the scrape-off layers (limiter shadow regions) of other tokamaks. ${ }^{3,4}$

The turbulence of the edge region is apparent in the large fluctuation level of $I^{+}$and floating potential $\phi_{f}$, typically $\tilde{I}^{+} / \bar{I}^{+}=e \tilde{\phi}_{f} / k T_{e} \cong 20 \%-50 \%$. In the present experiment we use only the average value of these quantities, which is well defined on a millisecond time scale.

\section{EXPERIMENTAL RESULTS}

\section{A. Basic effects}

The typical effects of a local toroidal divertor of strength $B_{C}= \pm 1.2 B_{T}$ on the plasma current, total uv emission, and line averaged density through the plasma center are shown in Fig. 3(a). It can be seen that these global quantities are not greatly changed by the activation of the divertor, at least over the time scale of this experiment. This absence of adverse global effects is an important prerequisite for operation of such a divertor, since in general such a nonaxisymmetric field perturbation may destory the magnetic flux surfaces (see Sec. IV B).

This type of a divertor is potentially useful for active control of global plasma properties such as density and impurity level, similar to the bundle divertor. ${ }^{17}$ However, the intention here is mainly to demonstrate the local control over the plasma-limiter interaction, and not to investigate the consequent global effects on the plasma.

The local effects of the divertor on the plasma-limiter interaction are determined by a scanning Langmuir probe as described in Sec. II B. In Fig. 3(b) are Langmuir probe ion saturation current signals $J^{+}$for the three discharges shown in Fig. 3(a), where for these cases the probe is fixed at a position $x=1 \mathrm{~cm}$ (i.e., $1.25 \mathrm{~cm}$ behind the leading edge of the limiter). The effects of the divertor can be seen by the decrease in $J^{+}$when the $B_{C}=-1.2 B_{T}$ field is applied, and by the increase in $J^{+}$when the $B_{C}=+1.2 B_{T}$ field is applied. A quantitative characterization of these effects on the local plasma flow is given in the next two sections.

Qualitatively, what happens is the following: for the $B_{C}=-1.2 B_{T}$ case the plasma flow to the limiter is decreased due to the diversion of the magnetic field away from the limiter, as shown in the field line plot in Fig. 3(b); similarly, for the $B_{C}=+1.2 B_{T}$ case the plasma field to the limiter is increased due to the diversion of the magnetic field into the limiter. Thus the plasma flow to the limiter, as monitored by the ion current to the probe adjacent to it, clearly shows the basic effects expected from the simple theoretical model described in the Introduction and in Sec. IV.

\section{B. Radial profiles of plasma flow to the limiter region}

In Fig. 4 are radial profiles of the ion saturation current density $J^{+}=I^{+} / A$ as measured by the movable limiter probe for the three cases $B_{C}= \pm 1.2 B_{T}$ and $B_{C}=0$ which were shown in the previous section. These profiles were taken $1 \mathrm{msec}$ after the activation of the divertor (and at this same time for the $B_{C}=0$ case). Each point represents one discharge in this data run.

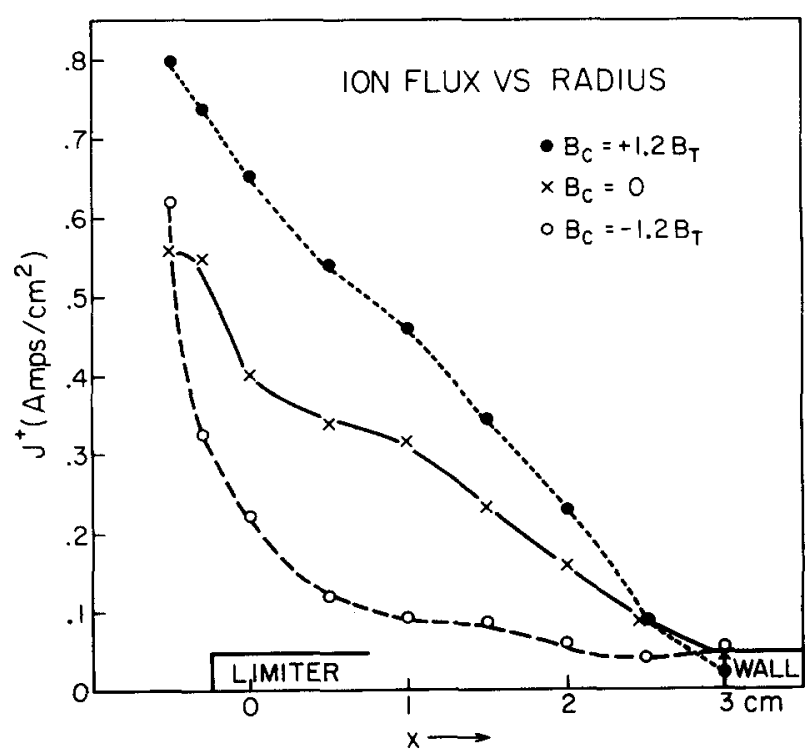

FIG. 4. Radial profiles of the probe ion saturation current $J^{+}$directly adjacent to the limiter, for the three cases shown in Fig. 3. The effect of the divertor is to increase the ion flux over most of the radial extent of the limiter for $B_{C}=+1.2 B_{T}$, and to decrease it over most of the radial extent for $B_{C}=-1.2 B_{T}$. These profiles were evaluated for a time near the peak of the divertor field. 
For the unperturbed case, $B_{C}=0$, the radial profile shows a monotonic decrease in $J^{+}$with radius over the whole extent of the limiter scrape-off region. This type of radial profile is similar to that observed in other tokamaks, ${ }^{3,4}$ and represents the result of cross-field diffusion from the confined plasma into the limiter's scrape-off region. The shape of this radial profile cannot as yet be predicted from theory, since the cross-field diffusion coefficients are not well known.

For the diverted cases, the radial profile of $J^{+}$was also observed to decrease monotonically with radius in the limiter scrape-off layer. However, when $B_{C}=+1.2 B_{T}$ the magnitude of $J^{+}$can be seen to increase over almost all of the radial extent of the limiter (with respect to the undiverted case), while when $B_{C}=-1.2 B_{T}$ the magnitude of $J^{+}$decreases over almost all of the limiter region.

A clearer picture of the effect of the divertor on the ion flux can be obtained by normalizing the $J^{+}$profiles with the divertor "on" to those taken with the divertor "off," again as evaluated approximately at the time of the coil's peak field.
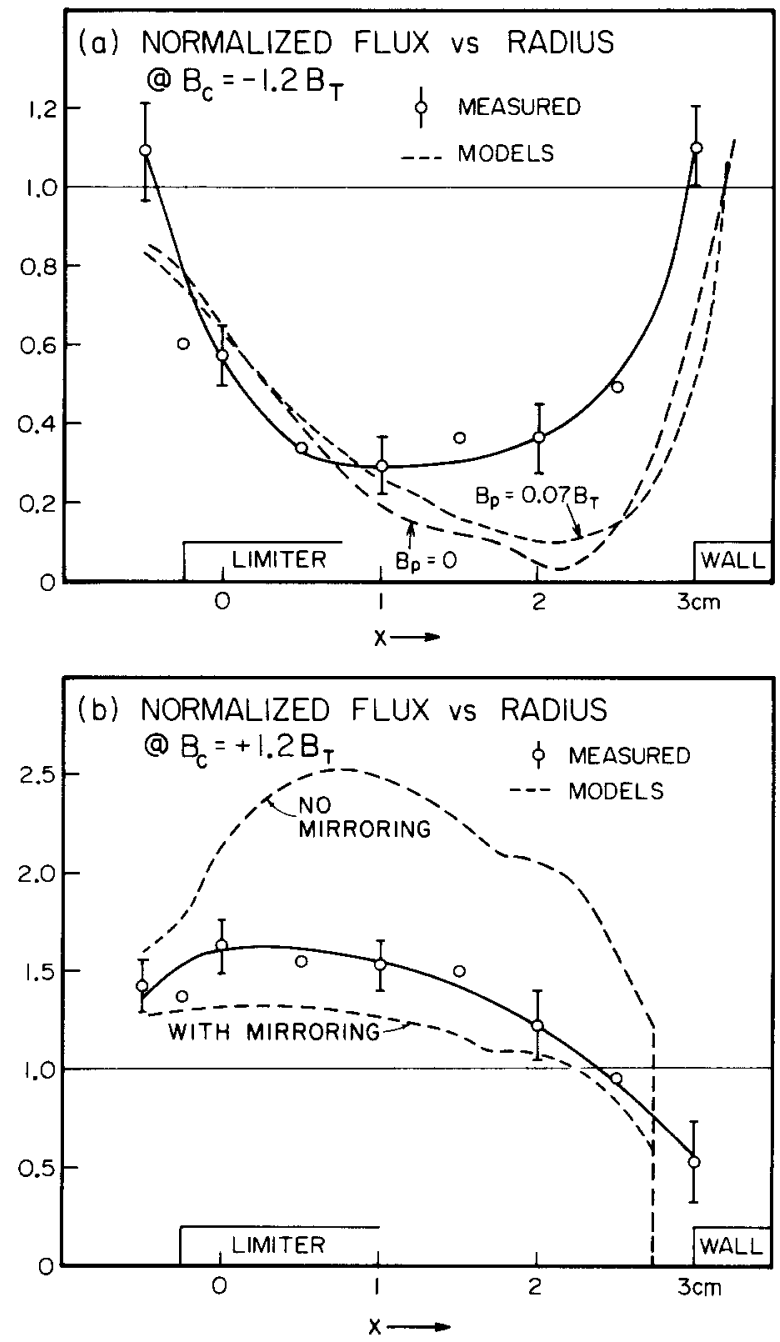

FIG. 5. Normalized radial profiles of $J^{+}$for the $B_{C}= \pm 1.2 B_{T}$ cases. For each point the value $J^{+}$obtained with the divertor "on" is divided by the value of $J^{+}$at the same point with the divertor "off," using data like that in Fig. 4. In (a) is the normalized radial profile for the $B_{C}=-1.2 B_{T}$ case, while (b) is the same but for $B_{C}=+1.2 B_{T}$. The dashed lines are derived from the theoretical model.
These normalized profiles are shown in Fig. 5 for the $B_{C}= \pm 1.2 B_{T}$ cases. The error bars in Fig. 5 span the results of four separate runs (including that of Fig. 4), each consisting of about 50 discharges, i.e., the effects shown in the figure are quite reproducible.

The normalized radial profiles of Figs. 5(a) and 5(b) display the main result of this experiment; namely, that the ion flux to the probe adjacent to the limiter can be either increased by at least $50 \%$, or decreased by at least a factor of 3 through use of a local toroidal divertor inside the limiter. These experimental results also show two other effects; namely, that the changes produced by the divertor are relatively weaker near the limiter's leading edge $(x \cong 0$ to $x=-0.25 \mathrm{~cm}$ ), and also that the direction of the change can be inverted near the wall. The shape of these profiles, including these two other effects, can be fairly well explained by the theoretical models (dashed lines in Fig. 5), as discussed in Sec. IV.

\section{Variation with $B_{C} / B_{T}$}

Results obtained by varying the magnitude of the divertor field $B_{C}$ are shown in Fig. 6. Here the toroidal field, limiter position, and plasma conditions were kept constant while $B_{C} / B_{T}$ (as measured at the coil center) varied from 0.8 to 1.8. The effects of the divertor were monitored at two radial positions, $x=0 \mathrm{~cm}$ near the leading edge and $x=1.5$ $\mathrm{cm}$ near the coil center. It was observed that the normalized values of $J^{+}$at a given probe position varied monotonically and approximately linearly with $B_{C} / B_{T}$, except perhaps in the range $B_{C} / B_{T}=+1.2-1.8$ in which the normalized flux was approximately constant. These results show that a significant effect on the local plasma flow in this limiter geometry can be obtained only with a strong local field perturbation, i.e., $B_{C} / B_{T} \sim 1$.

The expected behavior based on the theoretical model of Sec. IV A, shown in Fig. 6, is discussed in Secs. IV and V. Although the model is in reasonably good agreement with the data, a significant discrepancy occurs at the highest fields $B_{C} / B_{T} \leqslant-1.3$ at which the density near the coil center did not go to zero as predicted by the model (the model predicts zero flux at these fields because the probe should be inside the magnetic separatrix of the coil).

\section{Varlation with density and plasma positioning}

The results shown in Figs. 4-6 refer to discharges like those shown in Fig. 3 in which the density is $\bar{n}=4 \times 10^{12}$ $\mathrm{cm}^{-3}$ at the time when the coil current peaks. In Fig. 7 we show that these results are not particularly sensitive to the plasma density or to plasma positioning within the normal operation range of this machine. In this figure, the relative drop of $J^{+}$at $B_{C}=-1.2 B_{T}(1.5 \mathrm{~cm}$ behind the leading edge) is shown to be $0.3-0.4$, independent of the local density as varied by gas puffing over a nearly a factor of 10 . The results were also independent of the radial positioning of the plasma as varied by the vertical field (the density at the probe also changes somewhat with vertical field due to the in/out plasma movement). 

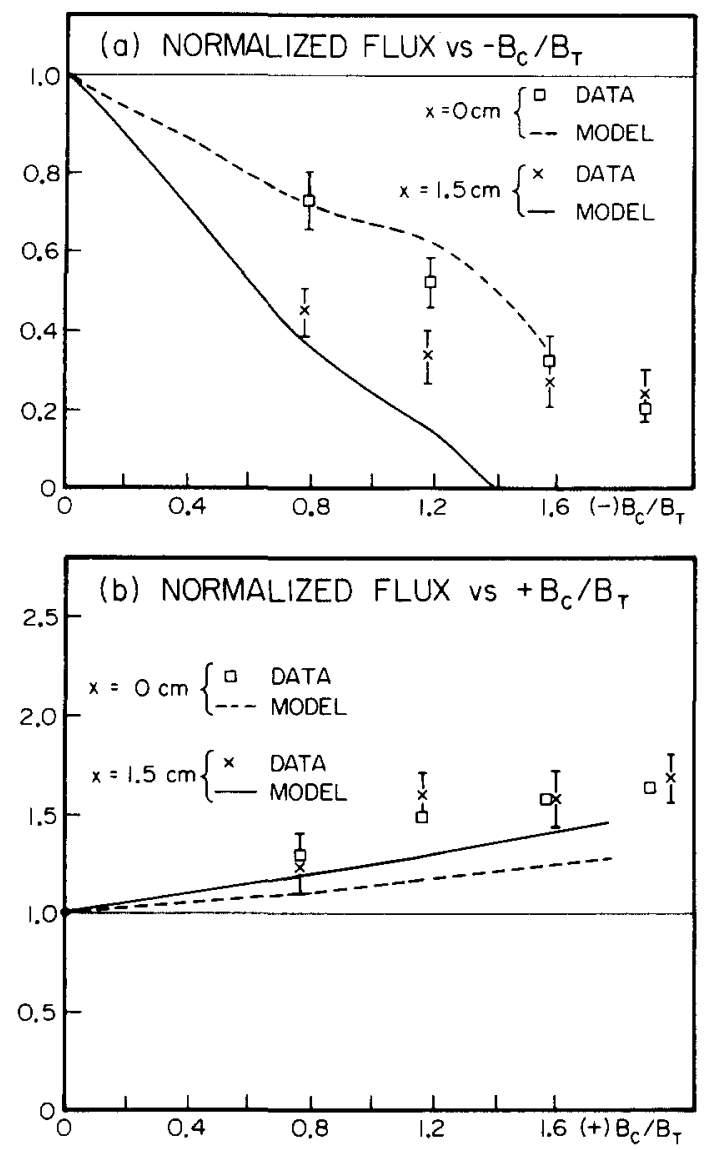

FIG. 6. Scaling of normalized flux at two radial positions versus relative strength of the divertor coil $B_{C} / B_{T}$. In (a) are the scalings for reversing field divertors, and in (b) are the same for increasing field divertors. Model calculations are also shown.

\section{E. Effects on temperature}

The effect of the local divertor on the electron temperature profile measured by the Langmuir probe adjacent to the limiter is shown in Fig. 8(a). These profiles were taken in the same type of discharges, and at the same time during the discharge, as the profiles of $J^{+}$shown in Fig. 4.

For the undiverted case $\left(B_{C}=0\right)$ there is a clear trend for $T_{e}$ in the scrape-off layer to fall from $20-25 \mathrm{eV}$ near the

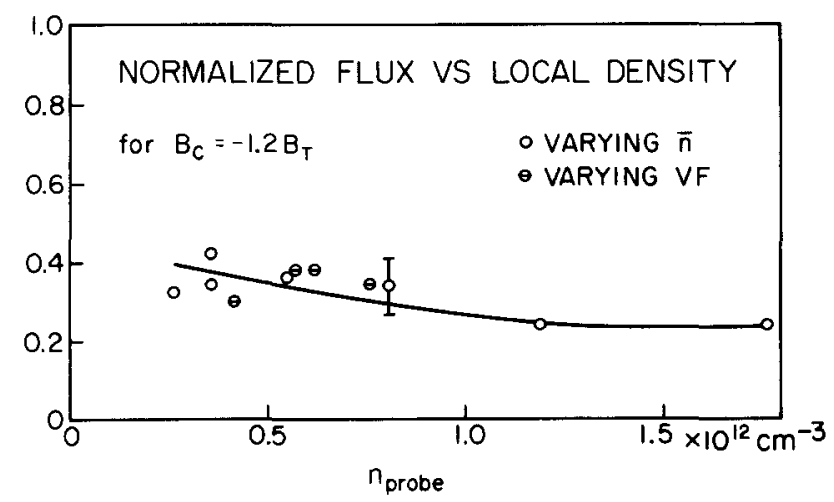

FIG. 7. Normalized flux for the $B_{C}=-1.2 B_{T}$ divertor as a function of the local density at the probe (as varied by gas puffing) and the radial in/out position (as varied by vertical field adjustment). The probe was located at $x=1.5 \mathrm{~cm}$ for all cases. The effect of the divertor is approximately independent of the local density and plasma positioning. (For Figs. 3-5, $n_{\text {probe }}=0.8 \times 10^{12} \mathrm{~cm}^{-3}$.) limiter's leading edge to $5-10 \mathrm{eV}$ near the wall. This $T_{e}$ profile is flatter than the corresponding $J^{+}$profile (Fig. 4), which shows a factor of 10 change over this same radial scan (however, if $J^{+}$were simply proportional to $n \sqrt{T_{e}}$, then the inferred density profile would be similar to the temperature profile). As before, there is as yet no readily applicable theoretical model to fit the scrape-off layer temperature profile, since the cross-field transport coefficients are not well known.

When the divertor fields are applied, the electron temperature profiles are observed to remain relatively unchanged. For example, in this case the temperature in the region $x=1-2 \mathrm{~cm}$ changes by less than $25 \%$ at $B_{C}=-1.2$ $B_{T}$, whereas in this same region the $J^{+}$changes by a factor of 3 for the same divertor field.

The predicted changes in $T_{e}$ based on the theoretical model are shown by the dashed lines of Fig. $8(\mathrm{~b})$, where the unperturbed temperature profile has been modeled by a linear temperature versus radius. The relative insensitivity of $T_{e}$ to the divertor can be explained (in part) by the fact that
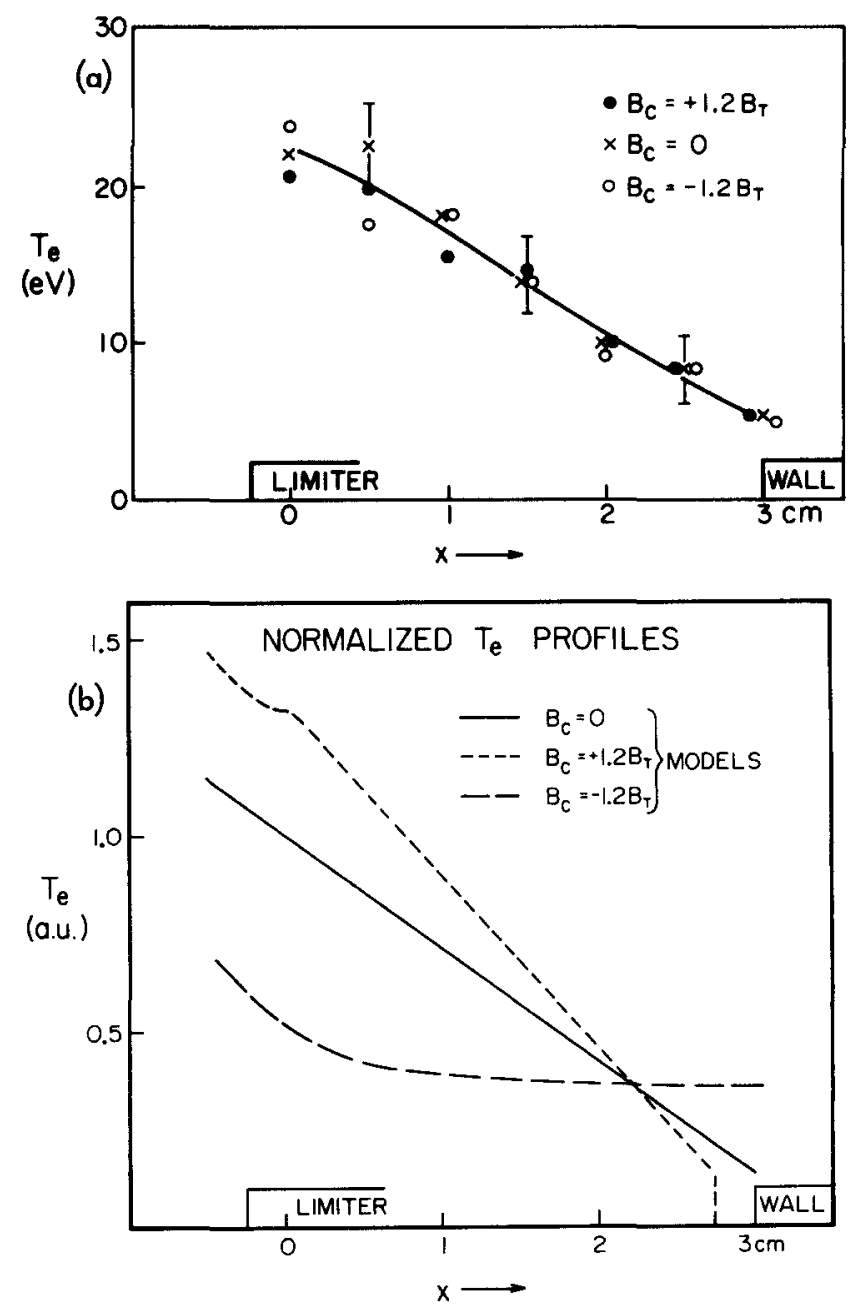

FIG. 8. Effects of the divertor on electron temperature profiles measured adjacent to the limiter under the same conditions as for Figs. 3-5. In (a) are the measured $T_{e}$ profiles with and without the divertor, and in (b) are the profile changes expected from the model. The expected flattening of the $T_{e}$ profile with the $B_{C}=-1.2 B_{T}$ divertor is not observed in the data. 
the expansion or contraction of the magnetic flux tubes and the mirroring should affect only the local density and not the temperature. However, there remains an effect on the $T_{e}$ profile expected due to the radial field line movement, as shown in Fig. 8(b), which is not seen in the data. This discrepancy is discussed in Sec. V B.

Since the temperature profiles are approximately invariant in the case corresponding to the data of Figs. 4 and 5, the observed changes in the $J^{+}$profiles in those figures are directly proportional to local density changes (i.e., the $T_{e}$ dependence of the relative $J^{+}$cancels out).
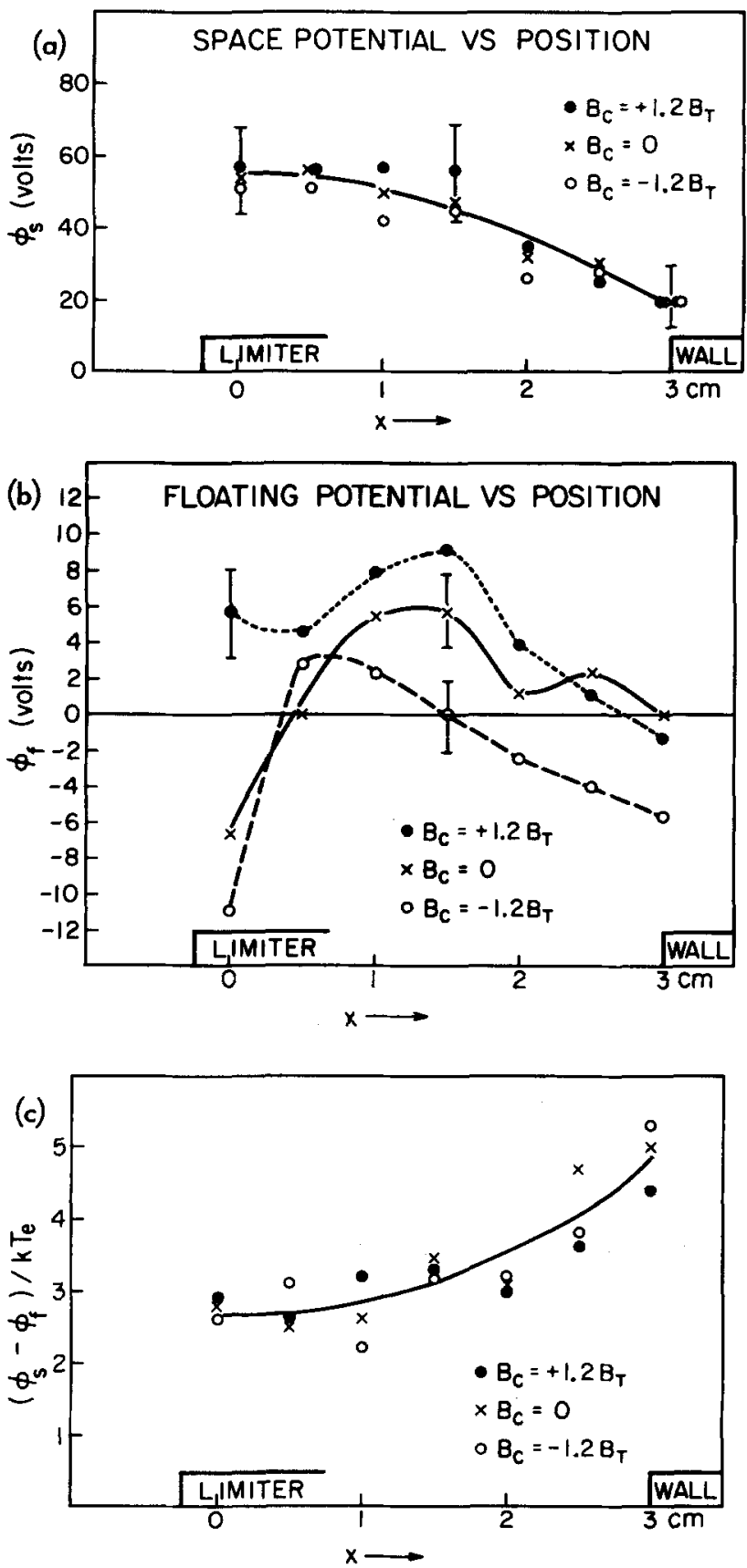

FIG. 9. Effects of the divertor on potentials measured adjacent to the limiter. In (a) are the space potentials, in (b) are the foating potentials, and in (c) are the calculated constants $c$ which relate these potentials to the electron temperature measurements of Fig. 8. The divertor has a small effect on the local floating potential.

\section{F. Effect on potentials}

In Fig. 9(a) is a plot of the radial profile of the space potential $\phi_{s}$ inferred from the "knee" of the same $(I, V)$ characteristics used to evaluate the $T_{e}$ profiles in Fig. 8(a). The discharges were of the same type used for Figs. 4, 5, and 8, and the data were taken at the time of peak divertor field as in the previous figures. The space potential is measured relative to the limiter, which is locally grounded to the chamber. Although there is considerable uncertainty in these values, they do indicate that the space potential of the plasma as measured at the probe is always positive, and that this potential is not dramatically changed by the application of the divertor fields at $B_{C}= \pm 1.2 B_{T}$.

However, a more precise measurement of potential changes can be made using the probe's floating potential $\phi_{f}$ instead of the potential. As shown in Fig. 9(b), there are small but systematic changes in $\phi_{f}$ which occur along with the divertor field activation. In particular, at $x=1-2 \mathrm{~cm}$ it can be seen that $\phi_{f}$ becomes a few volts more positive at $B_{C}=+1.2 B_{T}$ than at $B_{C}=0$, and a few volts more negative at $B_{C}=-1.2 B_{T}$ than at $B_{C}=0$. These changes occur in a region where the density is changing by $+50 \%$ and $-300 \%$, respectively. In all cases, however, the measured $\phi_{f}$ is within $\pm 10 \mathrm{~V}$ of the local limiter and chamber ground potential. It should be noted that similar changes in $\phi_{s}$ are not ruled out considering the error bars of Fig. 9(a).

In order to interpret these changes in $\phi_{f}$ we need to know the relationship between $\phi_{f}$ and the real potential of the plasma $\phi_{s}$. According to probe theory ${ }^{15,16}$ there should be a simple relationship between $\phi_{f}$ and $\phi_{s}$, namely $\phi_{f}=\phi_{s}-c k T_{e} / e$, where $c$ is some constant which depends on the ion/electron mass ratio (and possibly on the electron distribution function). If we assume that $T_{e}$ and $c$ are constant at a given point independent of the divertor field, then the changes in $\phi_{s}$, are equal to the changes in $\phi_{f}$, i.e., the points in the plasma at which the density is increased by the divertor become more positive, and vice versa. This result is discussed in Sec. V C.

The data of Figs. 8, 9(a), and 9(b) can be also used to find $c$ versus radius in the limiter shadow, as shown in Fig. 9(c). The resulting values of $c=2-5$ are in the range expected from probe theory for a hydrogen plasma. ${ }^{18,19}$

\section{G. Effects elsewhere in the scrape-off region}

Although the main concern of the present paper is to characterize the local effects at the limiter due to this divertor, a separate run was made to look for changes which might be occurring elsewhere in the limiter scrape-off region. This was done by measuring the ion saturation current drawn by a probe located $90^{\circ}$ away poloidally and $0^{\circ}$ toroidally from the limiter. At this position the probe was very far $(>5 \mathrm{~m})$ downstream along the field lines which intersected the limiter.

Results were obtained only for the case $B_{C}=-1.2$ $\times B_{T}$, as shown in Fig. 10. For $B_{C}=0$ the radial profile of $\mathrm{J}^{+}$at this position was similar to that obtained directly adjacent to the limiter (Fig. 4). However, for $B_{C}=-1.2 B_{T}$ there was not any significant change in the profile at this 


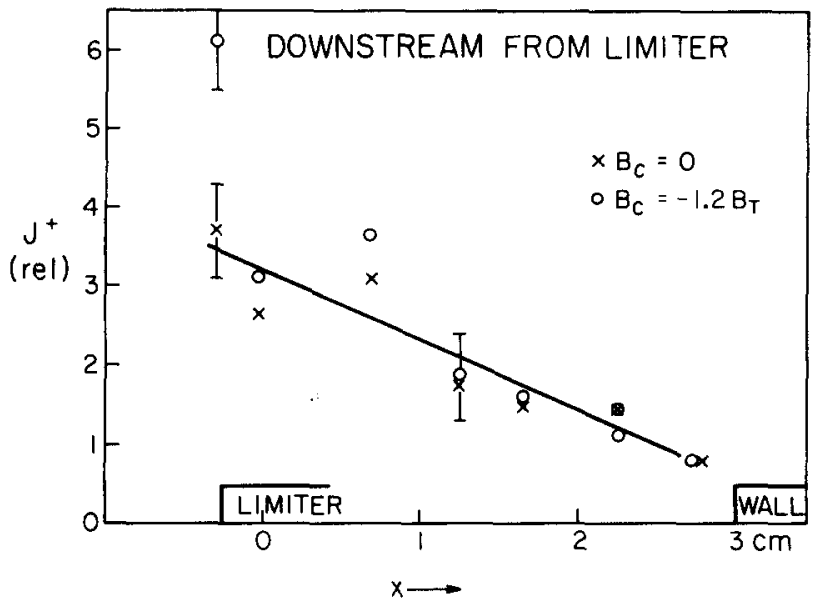

FIG. 10. Effect of the divertor $B_{C}=-1.2 B_{T}$ on the scrape-off layer profile far downstream toroidally from the divertor location. The divertor does not significantly affect the plasma edge profile in this region.

position, in contrast to the profile changes which were clearly observed adjacent to the limiter.

This result shows at least qualitatively that for distances sufficiently far downstream from the limiter, the scrape-off region is unperturbed by the divertor. This is reasonable since the local divertor mainly rearranges the magnetic structure near to the limiter, with the downstream effects eventually being smoothed out by cross-field diffusion. However, a quantitative evaluation of the downstream effects of the divertor was not attempted due to the limited access in this machine.

\section{MODEL MAGNETIC DIVERTOR CALCULATIONS AND COMPARISON WITH THE EXPERIMENTAL RESULTS}

\section{A. Theoretical model for divertor effects}

To help understand the observed effects of the divertor on the plasma flux to the probe directly adjacent to the limiter (Figs. 4-6), the following simple model was used: the radial profile of the plasma flux incident into the divertor region from downstream was assumed to be that measured by the probe when $B_{C}=0$ (as shown in Fig. 4). This was motivated by the fact that the global plasma properties were not significantly affected by the activation of the divertor, and by the fact that the measured radial profile at one point far downstream (Fig. 10) was not changed when the divertor was activated at $B_{C}=-1.2 B_{T}$. This plasma incident into the divertor region was then assumed to remain "frozen" to the field lines as the field lines approached the limiter, i.e., the incident radial profile was changed by the radial movement of the field lines near the limiter/divertor. Cross-field diffusion in the divertor region itself is negligible for $D_{\perp} \leqslant 10^{5} \mathrm{~cm}^{2} / \mathrm{sec}$ (generally the upper limit on diffusion in the edge region ${ }^{3}$ ).

Thus, the profile of the plasma flux as measured near the limiter would be affected by three things: (i) the extent to which the field lines are displaced radially as they approach the limiter, (ii) the fact that the magnetic flux tube of a field line will contract or expand as the magnetic field strength changes on entering the divertor region (leading to a com- pression or decompression of the plasma in the flux tube), and (iii) if the magnetic field strength increases as the field line approaches the divertor (which will be the case when the divertor field adds to the toroidal field), then the divertor also acts like a magnetic mirror, reflecting some of the incident plasma flux.

To implement this model, it is first necessary to calculate the trajectories of the magnetic field lines intersecting the limiter, and to then calculate the variation of magnetic field strength along these trajectories. To do this, the actual limiter coil shown in Fig. 1(b) is modeled as a rectangular loop, as shown in Fig. 2(a). The $x, y$, and $z$ directions correspond, respectively, to the radial, poloidal, and toroidal directions of the tokamak. The magnetic field due to the divertor coil, $B_{C}$, is calculated and added to the toroidal field $B_{T}$ (modeled as a constant field $B_{T}$ is the $z$ direction), so that the
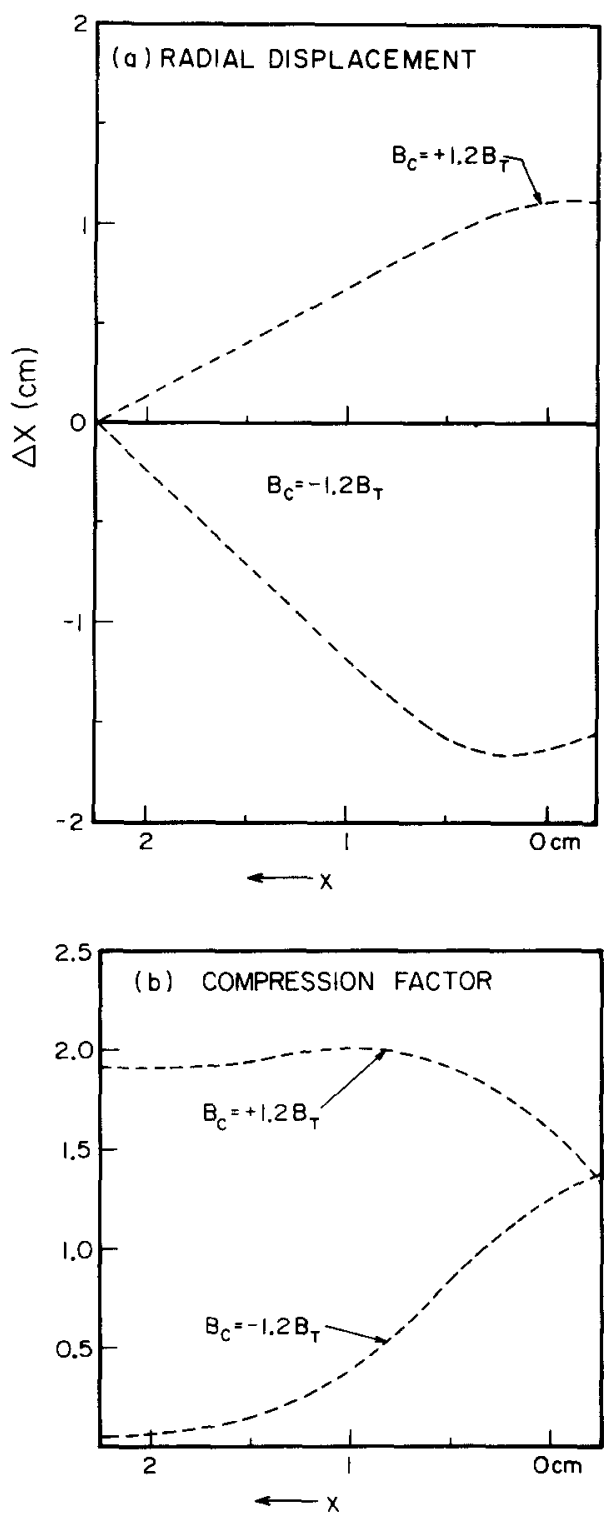

FIG. 11. Model calculations of the radial field line displacements and magnetic flux compression factors for the $B_{C}= \pm 1.2 B_{T}$ divertors. In (a) are the radial displacements for field lines which intersect the probe (with the divertor on) at the location specified by the $x$ coordinate; and in (b) are the magnetic flux compression factors specified similarly. 
trajectories of magnetic field lines in the total field are computed. The results of this calculation for a coil field strength $B_{C}= \pm 1.2 B_{T}$ are shown in Figs. 2(b) and 2(c). Effects on these trajectories due to the poloidal field, as modeled by a constant field in the $y$ direction, have also been included for some cases.

For such calculations, the change in the radial $(x)$ position of a field line between $z=\infty$ (far downstream) and $z=1.1 \mathrm{~cm}, y=0$ is found, where the latter is the line along which the ion flux is measured by the probe (see Fig. 2). These radial displacements, $\Delta x=x(z=1.1)-x(z=\infty)$, are plotted in Fig. 11(a) as a function of the field line position $x$ at $z=1.1, y=0$ for the magnetic configurations of Fig. 2 . Note that the $x$ axes of these figures are the positions at which the diverted field line intersects the probe. The field lines intersecting the geometric center of the coil $(x=2.2$ $\mathrm{cm}$ ) are not displaced at all, while the field lines which intersect the limiter near its leading edge $(x=0)$ have the largest radial displacement, as can be seen also in Fig. 2. In Fig. 11(b), the magnetic flux compression factor, i.e., the ratio of the total magnetic field strength as measured at the probe position $(z=1.1)$ divided by the magnetic field strength downstream $(z=t)$ is also plotted as a function of the field line position at $z=1.1, y=0$. It can be seen that for the case when $B_{C}=+1.2 B_{T}$ at the probe position the magnetic flux is increased by a factor $C \sim 2$, hence the plasma density should increase; while for the case when $B_{C}=-1.2 B_{T}$, the magnetic flux expands, $C<1$, over most of the limiter surface, and the plasma density should decrease.

The predicted plasma flux at a given probe position adjacent to the limiter should therefore be the plasma flux which is incident from downstream along that same field line, multiplied by the magnetic flux compression factor for that field line, i.e., $J^{+}(x)=C(x) J^{+}(x-\Delta x)$. The results of this calculation for $B_{C}=-1.2 B_{T}$ are plotted in Fig. 5(a), where the predictions of this model are compared to the measured plasma flux profile. In this figure both experimental data and the model calculation are normalized to the undiverted $\left(B_{C}=0\right)$ cases. The second theoretical curve in Fig. 5(a) includes in the model the effect of a constant poloidal $(\hat{y})$ field $B_{p}=0.07 B_{T}$. This small poloidal field has an influence when the toroidal field is nearly canceled by the coil field, which occurs near the separatrix. The fit of these theoretical curves to the data shows reasonably good agreement, as discussed in Sec. V A.

The effect of particle mirroring must also be included for cases when the coil field $B_{C}$ adds to the toroidal field [Fig. 5(b)]. The flux of particles through a mirror is reduced by the fraction $f=1 / M$, where $M=B(z) / B(\infty)$ is the mirror ratio, assuming the incident particle distribution function is isotropic. $^{20}$ Thus, the flux measured at $z=1.1$ should be reduced by a factor $f=B(z=\infty) / B(z=1.1)$ due to mirroring. However, as discussed above, the plasma on a given field line is also compressed by the same factor $C=1 / f$ due to the magnetic flux compression. Thus these two effects on the plasma flux to the limiter should exactly cancel. In Fig. 5(b), two theoretical curves are compared with the measured flux, one which includes this mirroring effect and one, labeled "without mirroring," which includes only the effects of mag- netic field compression and displacement. Clearly the agreement is better when the mirroring effect is included.

In Fig. 6, predictions of this model for the plasma flux at various ratios of $B_{C} / B_{T}$ are compared to the experimental results. For these calculations, a poloidal field of $B_{p}=0.07$ $\times B_{T}$ was included. The model shows fairly good agreement with the measurements over most of the range, as discussed in Sec. $\mathrm{V}$ A.

This theoretical model was also used to predict the effect of the divertor on the electron temperature profiles near the limiter. Again, the downstream $(z=\infty)$ temperature profile was assumed to be that measured at the probe adjacent to the limiter for the $B_{C}=0$ case, as shown in Fig. 8(a). Because of the rapid electron thermal conduction along a field line, the electron temperature was assumed to remain constant along a field line. Thus, the temperature near the limiter depends only on the radial displacement of the field lines and not on compression or mirroring. The predicted electron temperature profiles calculated for $B_{C}= \pm 1.2 B_{T}$ are shown in Fig. 8(b) for comparison with the measured profiles in Fig. 8(a). Possible reasons for the differences between the theoretical and experimental profiles are discussed in Sec. V B.

This simplified theoretical model neglects many effects which could also influence the plasma flux and temperature at the limtier in the presence of a magnetic divertor. The possible effects of finite ion gyroradius size, atomic physics processes, ${ }^{21,22}$ nonisotropic distribution functions, electrostatic potentials, and enhanced cross-field diffusion near the divertor field nulls are discussed in Sec. $V$.

\section{B. Divertor effects on flux surfaces}

The above theoretical model dealt only with the local effects of the toroidal divertor on the plasma flux and temperature near the limiter. It is also important to know whether the local magnetic perturbation will significantly affect the plasma globally through changes in the quality of the interior magnetic flux surfaces. For example, such changes are important for optimizing the design of bundle divertors, which aim to leave unperturbed the interior flux surfaces; on the other hand, there are other magnetic limiter designs which aim to "ergodize" the magnetic structure of the edge plasma in order to control the plasma-wall interaction.

An Oak Ridge magnetic field line tracing code ${ }^{23}$ was used to map out flux surfaces for typical divertor configurations of this experiment, as shown in Fig. 12. For these cases it can be seen in $12(\mathrm{~b})$ and $12(\mathrm{c})$ that at the level $B_{C}= \pm 1.2$ $B_{T}$ the interior flux surfaces $(r / a \leqslant 0.7)$ are not significantly perturbed, so that global plasma confinement ought not to be degraded. However, for the cases $12(\mathrm{~d})$ and $12(\mathrm{e})$ in which $B_{C}= \pm 2.0 B_{T}$ some radial wandering from the unperturbed flux surfaces can be seen, and in fact some of the field lines which initially intersect the limiter in these cases have been observed to wander into the wall before completely mapping out a flux surface.

These calculations show that a small, localized toroidal divertor such as this can leave most of the interior magnetic structure unperturbed, a result which is consistent with the 


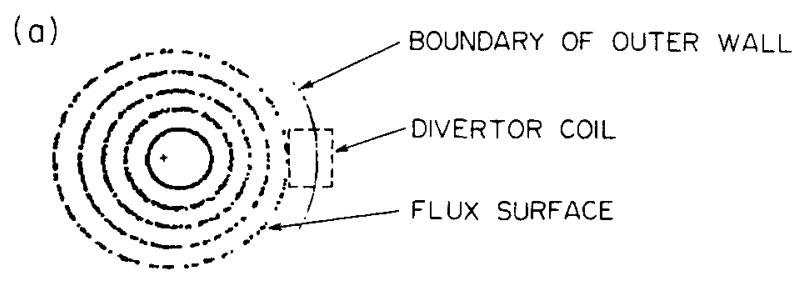

(b)
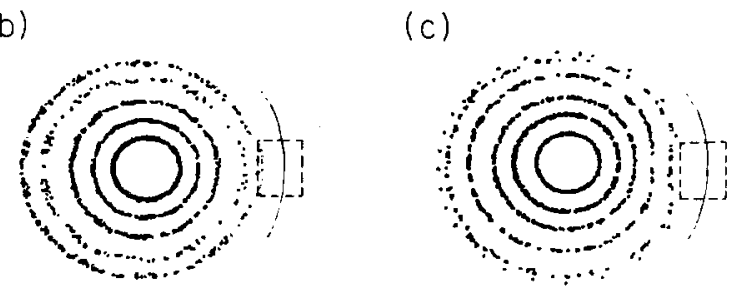

(d)
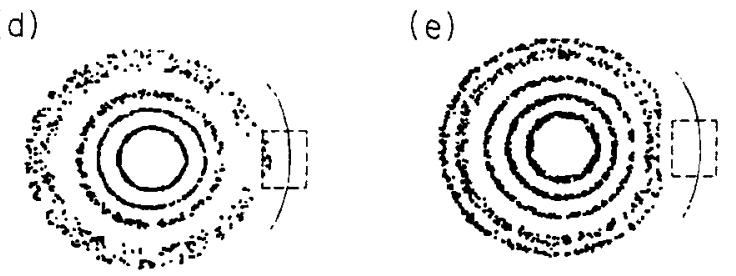

FIG. 12. Puncture plots of field line intersections with the poloidal plane which contains the divertor coil, with the coil indicated by the dashed box at the right. The divertor strengths are (a) $B_{C}=0$, (b) $B_{C} / B_{T}=+1.2$, (c) $B_{C} / B_{T}=-1.2$, (d) $B_{C} / B_{T}=+2.0$, and (e) $B_{C} / B_{T}=-2.0$. The interior flux surfaces are not seriously perturbed at the levels shown in (b) and (c), which correspond approximately to the usual experimental configuration.

experimental observations of the lack of global effects of this divertor at $B_{C}= \pm 1.2 B_{T}$. These calculations also show that the exterior flux surfaces can be perturbed at this level. However, these disturbances of the exterior flux surfaces should not have a significant influence on the local magnetic field model described in Sec. IV A, since this perturbed flux surface structure would be evident only after many toroidal transits, and so may only affect the profile of plasma flux incident into the divertor region.

\section{DISCUSSION}

\section{A. Fit of divertor model to ion flux results}

The simple divertor model of Sec. IV isolated three effects which should dominate the flow pattern of plasma to this local toroidal divertor: the radial movement of the field lines, the expansion or contraction of the flux tubes as they approach the limiter, and the particle mirroring. In this section we discuss the degree to which this model fits the experimental results. Where there is a discrepancy, some additional effects which may explain the observed behavior are discussed.

Returning to the $B_{C}=-1.2 B_{T}$ divertor case shown in Fig. 5(a), we see that a reduction in plasma flux to most of the radial extent of the limiter region $(x=0-2.5 \mathrm{~cm})$ was predicted by the model; in particular, the agreement in the region $x=0-1 \mathrm{~cm}$ is within the experimental uncertainties. On the other hand, the model significantly underestimates the ion flux to the region around $x=2 \mathrm{~cm}$, which is the region where the flux expansion factor dominates the expected changes [Fig. 11(b)]. This discrepancy may be related to the fact that in the region near $x=2 \mathrm{~cm}$ the toroidal magnetic field is nearly canceled out $(C \cong 0.1)$, hence the ion gyroradii of the particles incident along these field lines is large enough so that the ions can be found a significant distance away from their guiding center field line (for $B=0.2 \mathrm{kG}$ and $T=20 \mathrm{eV}, \rho_{i}=3 \mathrm{~cm}$ ). This effect would tend to radially average the profiles at the limiter similarly to the profiles observed in the data. Another possibility is that there is an enhanced cross-field diffusion in the near-zero field region, which would tend to fill the expected minimum in the radial profile around $x=2 \mathrm{~cm}$. A local cross-field diffusion coefficient on the order of $D_{1} \cong 10^{7} \mathrm{~cm}^{2} / \mathrm{sec}$ would be needed to produce this effect. This is much higher than the Bohm-type diffusion evaluated in the unperturbed toroidal field, $D_{\perp} \leqslant 10^{5} \mathrm{~cm}^{2} / \mathrm{sec}$.

The lessened effect observed experimentally near the limiter's leading edge $(x=0)$ in Fig. 5(a) can be identified as being due to relatively less flux expansion there, even though the radial displacement of the field lines is large (see Fig. 11). This is a general feature of such divertor geometries, since at the coil segment itself the toroidal field is not canceled significantly.

The lessened effect observed experimentally near the wall in Fig. 5(a) is also at least qualitatively explained by the model. This is simply due to the reversed radial movement of the field lines for $x>2.2 \mathrm{~cm}$, which causes a higher density part of the unperturbed profile to be pulled outward near the limiter, as shown in Fig. 2(c).

Turning to the $B_{C}=+1.2 B_{T}$ data shown in Fig. 5(b), we see that the increase in ion flux to most of the limiter region is reasonably well predicted by the model with mirroring. In particular, the inversion of the profile for $x>2.2$ $\mathrm{cm}$ is at least qualitatively explained by the reversal of the direction of radial field line movement, which connects this region to the outer wall [see Fig. 2(a)]. The small discrepancy between the calculated curve with mirroring and the data may be due to a nonisotropy in the ion distribution function which could affect the fraction of ions mirrored. (The distribution functions are not known in this experiment.)

The results of Fig. 6 show how the model predictions and experimental data scale with $B_{C} / B_{T}$ for two points on the profile, $x=0 \mathrm{~cm}$ (near the leading edge) and $x=1.5 \mathrm{~cm}$ (near the coil center). As shown in Fig. 6(a), within the range $B_{C} / B_{T}=-0.8$ to -1.6 the model fits the data at $x=0$ $\mathrm{cm}$ to within the experimental uncertainties. However, for the $x=1.5 \mathrm{~cm}$ cases the model significantly underestimates the flux observed, as described for the $B_{C}=-1.2 B_{T}$ case above. In particular, for $B_{C} / B_{T}<-1.3$ the model predicts that zero flux should appear at $x=1.5 \mathrm{~cm}$, since the probe then lies within the separatrix surrounding the coil. Since there is definitely a nonzero ion flux at these fields even up to $B_{C} / B_{T}=-1.75$, some other effects such as those discussed in the second paragraph of this section must be involved.

The scaling results in Fig. 6(b) for increased field show a reasonably good agreement with the model including mir- 
roring, except for a small systematic underestimate of the observed ion flux magnitude. The data also seem to show a saturation in the observed effect for $B_{C} / B_{T}=+1.2-1.7$. In practice, this may mean that it would be difficult to increase the ion flow to the limiter by more than a factor of about 1.6 in this configuration.

The density scaling results of Fig. 7 are again consistent with the model in that the relative effect of the divertor at a fixed $B_{C} / B_{T}$ should not depend on the particular value of density at the limiter, assuming the profiles in the scrape-off layer are unchanged with density. The slight increase which is observed in the divertor's effect at the highest densities may be due to such a change in the shape of the incident radial profile with density.

Overall, we conclude that the local effects of the divertor on flux profiles at the limiter can be reasonably well predicted by the simplified magnetic field model described in Sec. IV A. The biggest discrepancies occur at high reversing fields, where perhaps the large ion gyroradii or enhanced diffusive effects are important.

\section{B. Comparison of model to temperature data}

The electron temperature data of Fig. 8(a) shows that no systematic changes in any part of the $T_{e}$ profile were seen for the cases $B_{C}= \pm 1.2 B_{T}$. In Fig. 8(b) are shown the changes which were expected from the model due to radial movement of the unperturbed temperature profile (see Sec. IV A). A comparison of Figs. 8(a) and 8(b) shows that the expected effects of the divertor were not obtained; in particular, the flattening of the profile for $B_{C}=-1.2 B_{T}$ should have been observed despite the error bars on the $T_{e}$ measurement.

One possible explanation for this discrepancy would involve systematic changes in the incident downstream temperature profile caused by the divertor, which could compensate for the expected effect due to the field line movement. Although such changes cannot be ruled out, they would have to be such as to result in a nearly invariant temperature profile. Another explanation would be that the temperature profile at the limiter is determined not by the temperature profile incident from downstream along the field line, but rather by processes not dependent on the magnetic field, i.e., those involving neutrals. ${ }^{21,22}$ For example, the electron temperature could be locally determined by radiative losses proportional to the local neutral density at various points along the limiter. However, since the local neutral density at the limiter is not measured, and since modeling of even the unperturbed scrape-off layer temperature profile is very difficult, ${ }^{4}$ we cannot yet give a quantitative explanation for the behavior of these profiles within the divertor region.

\section{Plasma potential}

The simplified magnetic field model of Sec. IV A does not include effects relating to the plasma electrostatic potential. However, it is well known for magnetic mirrors and bundle divertors ${ }^{24,25}$ that variations of potential along a magnetic field line can exist and can be important in the description of plasma flows. Specifically, if electrons obey a Boltzmann distribution along a field line, and if quasineutrality is assumed (outside the Debye sheath), then the electro- static potential can be written

$$
\delta \phi=\phi(z)-\phi(\infty)=k T_{e} \ln [n(z) / n(\infty)],
$$

where $n(z)$ and $\phi(z)$ are the density and potential at some point along the field line, and $n(\infty)$ and $\phi(\infty)$ are the density and potential at a reference point taken to be at $z=\infty$.

This equation can be applied most simply to our situation by considering the field line which initially passes through the center of the coil at $x=2.2 \mathrm{~cm}$, since at this point the field line is not moved when the divertor fields are activated. If for $B_{C}= \pm 1.2 B_{T}$ we assume that the density far downstream $(z=\infty)$ along this field line is given by the density measured at the probe for $B_{C}=0$, and that the density is proportional to the measured ion saturation current, then the expected change in potential due to the divertor at $x=2.2 \mathrm{~cm}$ should be determined through Eq. (1) by the local electron temperature and by the relative effect of the divertor on the measured ion saturation current at this point.

Referring to Figs. 5 and 8 for the normalized flux ratios and electron temperatures at $x=2.2 \mathrm{~cm}$, we find that the expected potential changes are $\delta \phi=k T_{\mathrm{e}} \ln (0.4) \cong-8 \mathrm{~V}$ for $B_{C}=-1.2 \quad B_{T}$, and $\delta \phi=k T_{e} \ln (1.1) \approx+1 \quad \mathrm{~V}$ for $B_{C}=+1.2 B_{T}$. These values are comparable to the measured floating potential changes of about $-5 \mathrm{~V}$ and $+1 \mathrm{~V}$, respectively, as shown in Fig. 9(b). However, only a qualitative agreement between expected and measured potentials can be suggested, since the uncertainty of $\pm 10 \mathrm{eV}$ in the measured temperatures comes into play both through Eq. (1) and through the conversions of the floating potential to the plasma space potential.

Although a quantitative evaluation of the potential changes cannot be made, such changes might be important in determining ion flow into the divertor region. For example, if when $B_{C}=-1.2 B_{T}$ the potential in the divertor region becomes negative with respect to its downstream value, then the parallel electric field might accelerate additional ions into the divertor region or change their distribution function to increase the flux to the limiter in our geometry.

\section{Plasma fiow to the limiter}

The discussion up to this point has been solely concerned with the interpretation of the measurements made by the probe directly adjacent to the limiter. However, there are two additional effects which need to be considered when calculating the plasma flow to the limiter itself.

First is the simple point that the probe measures the ion flux parallel to the field line, whereas the flux into the limiter also depends on the angle of the limiter surface with respect to the field line. For example, in the $B_{C}=-1.2 B_{T}$ case shown in Fig. 2(c) the field lines near the leading edge if the limiter $(x=0)$ intersect it at an angle of approximately $45^{\circ}$, therefore the local particle flux into the limiter surface itself (per unit area) will be reduced by about $40 \%$ from the value measured by the probe at that location.

The other effect is more complex and concerns the potential of the limiter. If the limiter potential were such as to collect all incident ions, then the ion flux to it should be the same as that measured by the local probe's ion saturation current. This situation would be expected for a floating li- 
miter (zero net current), since the sheath which develops makes the limiter negative with respect to the incident plasma. In the present experiment the limiter was grounded, but since the local space potential of the plasma was measured to be positive with respect to the limiter, the limiter again would be expected to collect all of the ions measured by the adjacent probe.

However, if for some reason the potential of the limiter was positive with respect to the plasma, then the ion flow to it would be impeded. This is unlikely to occur under normal conditions, but could be done intentionally by external biasing.

Furthermore, when considering the heat flux to the limiter the electrons contribute as well as the ions, and the effects of potentials could become particularly important. The effects of potentials are presently difficult to measure (see V C), and the heat flux effects, although important for practical applications, are beyond the scope of the present paper.

\section{CONCLUSIONS AND EXTRAPOLATIONS FOR FURTHER APPLICATIONS}

\section{A. Conclusions}

A method for locally controlling the plasma flow to a tokamak limiter has been described. In our configuration a small divertor coil mounted within an outer limiter produced a local magnetic field, comparable in strength to the toroidal field, which significantly redistributed the plasma flow to the limiter region. A simple magnetic field model for this effect was shown to fit the observed ion flux measurements fairly well, although some discrepancies and possible explanations for them were also discussed.

Specific conclusions include the following.

(1) Ion flux to most of the limiter region could be decreased by a factor of 3 or increased by $50 \%$ by application of a local divertor field which either subtracts from or adds to the local toroidal field.

(2) These effects on plasma flow to the limiter region could be obtained without greatly affecting global plasma confinement or impurity levels.

(3) A simple theoretical model which included radial field line movement, expansion or contraction of the flux tubes, and particle mirroring could explain the basic patterns of plasma flow to the limiter region.

(4) Two effects were observed which were not consistent with the model; namely, the enhanced plasma flow across the local separatrix for the reversing field divertor, and the invariance of the electron temperature profile with the application of the divertor.

\section{B. Extrapolation for further application}

In Fig. 13 we show two configurations in which magnetic control of the plasma-limiter interaction may be applied to pumped limiter designs. In Fig. 13(a) is a schematic drawing of a "scoop"-type pumped limiter similar to those used recently on moderate-sized tokamaks, ${ }^{7}$ in which a coil geometry similar to ours (dashed line) may be used to control plasma flow to the pump duct (shaded area). A particularly (a)

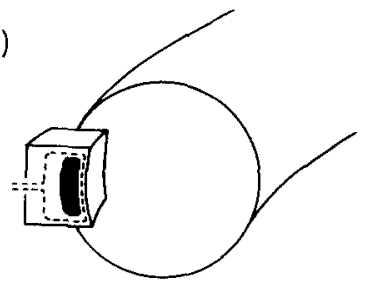

(b)

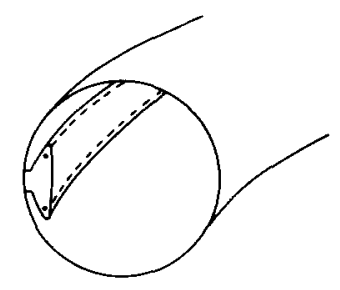

FIG. 13. Possible uses of local divertors for pumped limiter applications. In (a) is a toroidal divertor similar to that in the present experiment, showing the divertor coil by a dashed line and the pumping port by a black hole; and in (b) is a local poloidal divertor coil, shown by the dashed line, mounted inside a toroidal belt limiter. interesting case would be obtained if the leading edge of the limiter could become enclosed inside the coil's separatrix at $B_{C} \gg+B_{T}$, so that this edge would be isolated from the downstream plasma at the same time that the plasma is diverted and compressed into the pump duct.

In Fig. 13(b) a poloidal-type local divertor coil is shown mounted into the body of a toroidal pumped limiter similar to that designed for future large tokamaks. In this case the divertor coil (dashed line) can form a local separatrix around the limiter's leading edge to protect it from overheating, while at the same time the perturbed field can direct particles behind the limiter into the pumping ports. The coil current required for such a local diversion would be less than those required for a normal poloidal divertor, since the separatrix could be localized very near the coil. A significant control over the plasma flow could be obtained even if the separatrix was located inside the limiter body.

\section{ACKNOWLEDGMENTS}

We thank J. A. Rome, R. Fowler, V. Lynch, and M. Peng for help in using the Oak Ridge National Laboratory magnetic field line codes.

This work was supported by the U.S. Department of Energy, under Contract No. DE-AS03-SF00767.

'J. A. Schmidt, Comments Plasma Phys. Contolled Fusion 5, 225 (1980).

${ }^{2}$ R. E. Slusher and C. M. Surko, Phys. Rev. Lett. 40, 400 (1978).

${ }^{3}$ S. J. Zweben and R. J. Taylor, Nucl. Fusion 23, 513 (1983).

${ }^{4}$ P. Staib, J. Nucl. Mater. 111 \& 112, 109 (1982).

${ }^{5}$ A. Hasegawa and M. Wakatani, Phys. Rev. Lett. 50, 682 (1983).

${ }^{6}$ B. A. Carreras, P. W. Gaffney, H. R. Hicks, and J. D. Callen, Phys. Fluids 25, 1231 (1982).

${ }^{7}$ S. Talmadge, R. W. Conn, A. K. Prinja, and R. J. Taylor, Nucl. Fusion 22, 1369 (1982).

${ }^{8}$ D. O. Overskei, Phys. Rev. Lett. 46, 177 (1981).

${ }^{9}$ A. Jacobsen, Nucl. Fusion 22, 277 (1982).

10P. Mioduszewski, J. Nucl. Mater. 111 \& 112, 253 (1982).

${ }^{1}$ S. A. Cohen, R. Budny, G. M. McCracken, and M. Ulrickson, Nucl. Fusion 21, 233 (1981).

${ }^{12}$ S. J. Zweben, P. C. Liewer, and R. W. Gould, J. Nucl. Mater. 111 \& 112, 39 (1982).

${ }^{13}$ L. Oren and R. J. Taylor, Nucl. Fusion 17, 1143 (1977).

${ }^{14}$ S. Zweben and R. J. Taylor, Plasma Phys. 23, 337 (1981). 
${ }^{15}$ F. F. Chen, in Plasma Diagnostic Techniques, edited by R. Huddlestone and S. Leonard (Academic, New York, 1965), p. 113.

${ }^{16}$ M. J. M. Parrot, L. R. O. Storey, L. W. Parker, and J. G. Laframboise, Phys. Fluids 25, 2388 (1982).

${ }^{17}$ S. K. Erents, S. J. Fielding, R. D. Gill, D. H. J. Goodall, P. J. Harbour, J. Hugill, G. M. McCracken, J. E. Partridge, J. W. M. Paul, B. A. Powell, R. Prentice, G. Proudfoot, A. D. Sanderson, J. H. Shea, C. J. Sofield, D. D. R. Summers, J. E. Vince, and A. J. Wootton, in Proceedings of the 8th International Conference on Plasma Physics and Controlled Nuclear Fusion Research, Brussels, 1980 (IAEA, Vienna, 1980), p. 697.

${ }^{18}$ G. A. Emmert, R. M. Wieland, A. T. Mense, and J. N. Davidson, Phys.
Fluids 23, 803 (1980).

${ }^{19}$ R. Chodura, Phys. Fluids 25, 1628 (1982).

${ }^{20}$ L. Spitzer, Physics of Fully Ionized Gases (Wiley, New York, 1962), p. 14.

${ }^{21}$ D. R. Baker, R. T. Snider, and M. Nagami, Nucl. Fusion 22, 807 (1982).

${ }^{22}$ M. Petravic, D. Heifetz, D. Post, W. Langer/and C. Singer, in Proceedings of the 9th International Conference on Plosma Physics and Controlled Nuclear Fusion Research, Baltimore, 1982 (IAEA, Vienna, 1983), p. 323.

${ }^{23}$ R. H. Fowler, D. K. Lee, P. W. Gaffney, and J. A. Rome, Oak Ridge National Laboratory Report ORNL/TM-6293, June 1978.

${ }^{24}$ A. Nicolai, J. Nucl. Mater. 93 \& 94, 231 (1980).

${ }^{25}$ A. W. Bailey and A. A. Emmert, J. Nucl. Mater. 111 \& 112, 403 (1982). 